\title{
Automated Ultrasonic Measurement of Fetal Nuchal Translucency Using Dynamic Programming
}

\author{
Yu-Bu Lee ${ }^{1}$ and Myoung-Hee Kim ${ }^{1,2}$ \\ ${ }^{1}$ Department of Computer Science and Engineering \\ Ewha Womans University, Seoul, Korea \\ basilia@ewhain. net \\ ${ }^{2}$ Center for Computer Graphics and Virtual Reality \\ Ewha Womans University, Seoul, Korea \\ mhkimeewha.ac.kr
}

\begin{abstract}
Measurement of nuchal translucency (NT) thickness in the first trimester of pregnancy has recently been proposed as the most useful marker in the early screening for fetal chromosomal abnormalities. Ultrasonic measurement of NT thickness is currently performed by manually tracing the two echogenic lines and locating the electronic calipers on the inner edges of these lines. The drawbacks of this method are inter- and intra-observer variability, and its inefficiency. In particular, accurate caliper placement requires highly skilled operators since the border of the nuchal translucency layer is very thin. We present a computerized method of detecting the border of the NT layer by minimizing a cost function using dynamic programming. Local measurements of intensity, edge strength and continuity are extracted and become weighted terms in a cost function. Our method can obtain accurate and reproducible results, and has been validated by computing the correlation coefficient between manual and automatic measurements.
\end{abstract}

\section{Introduction}

Ultrasonography (US) is performed during early pregnancy for dating, determination of the number of fetuses, assessment of early complications, and increasingly for evaluation of the fetus, including measurement of the nuchal translucency (NT) thickness [1]. Measurement of NT thickness in the first trimester of pregnancy has proved to be one of the most discriminating prenatal markers of screening for chromosomal abnormalities such as trisomies 13, 18 and 21 [2]. Furthermore, an increased NT thickness in the presence of a normal karyotype is associated with an increased frequency of structural defects and genetic syndromes [3]. The NT thickness is measured in the sagittal section of the fetus by transabdominal or transvaginal ultrasound examination. Measurement is obtained manually by placing the calipers on the inner edges of the two thin echogenic lines that border the nuchal translucency layer. The crossbar of the caliper should be positioned so that one of its lines is hardly visible as it merges with the white line of the border. This not only requires highly skilled operators but it is also prone to inter- and intra-observer variability. There has been a little research on the computerized automation of 
fetal NT measurement. However, Bernardino et al. developed a semiautomated computerized measurement system, which uses the Sobel operator to detect the border of the NT layer [4]. In conventional edge-detection, the location of an edge is entirely determined by local evaluation of a single image feature such as the intensity or the intensity gradient. But a single image feature is not sufficient for reliable border measurement in fetal ultrasound images. These images frequently include weak echoes, echo dropouts, and speckle noise which makes them difficult to analyze using conventional border-detection techniques. Gustavsson et al. [5-7] have suggested a global optimization approach based on dynamic programming (DP) for detecting the boundaries of carotid artery which takes multiple image features into account. This method has obtained accurate and reproducible results for automated ultrasonic measurement by considering multiple image features such as intensity, gradient and border continuity.

In this paper, we will present a procedure based on dynamic programming to measure NT thickness automatically. We start by defining a region of interest in order to reduce the interference from the image boundary and to adapt to different fetal head positions and sizes of the NT layer. We then apply a dynamic programming procedure to determine the location of the border of the NT layer in a way that minimizes a cost function. This cost function is a weighted sum of terms that include local measurements of the echo intensity and the intensity gradient of the image, and a geometrical constraint on the shape of the borders. Our method overcomes the limitations of ultrasound images and accurately detects the border of the NT by combining multiple image features. Moreover, we avoid the variability of manual measurements.

The rest of this paper is organized as follows. In Section 2, we describe the characteristics of nuchal translucency images, definition of the region of interest, the segmentation procedure for detecting the border of the NT layer, and the automated measurement procedure. The results of this method are evaluated in Section 3, and then we draw some conclusions in Section 4.

\section{Methods}

In this section, we describe the characteristics of nuchal translucency images, the definition of the region of interest and the border detection method, and then we explain the automatic measurement procedure.

\subsection{Nuchal Translucency}

Nuchal translucency (NT) refers to the normal subcutaneous fluid-filled space between the back of the neck of a fetus and the overlying skin [8]. Fig. 1 shows a representative image of the NT and a schematic illustration of the echo zones (Z1-Z5) and the borders (B1, B2) of the NT layer. The NT thickness is defined as the maximum thickness of the translucent space (Z3) between the skin (Z4) and the soft tissues (Z2) overlying the cervical spine in the sagittal view of the fetus. 

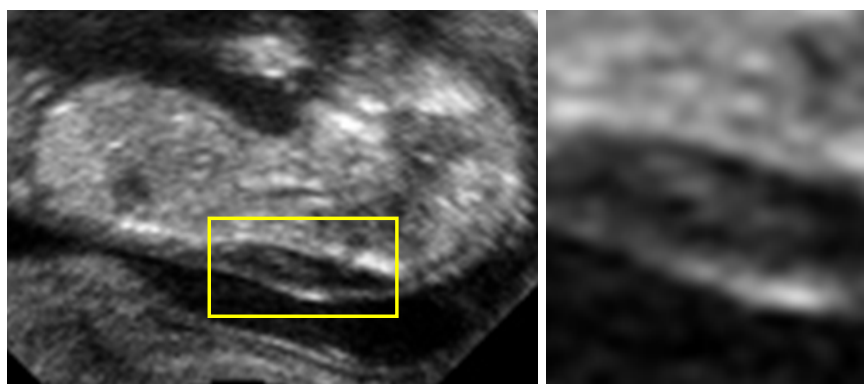

Echo zones

Z1

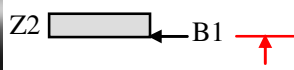

Z3 NT thickness

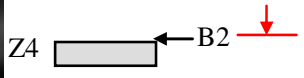

Z5

Fig. 1. Echo zones and NT borders

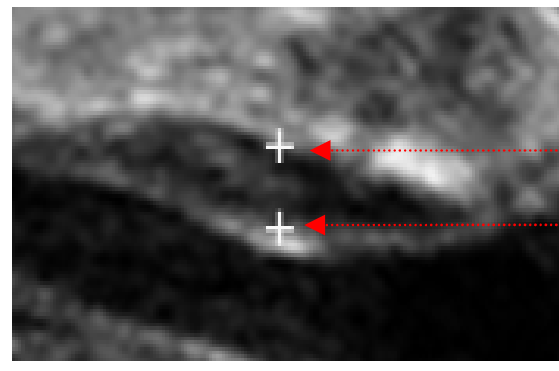

(a)

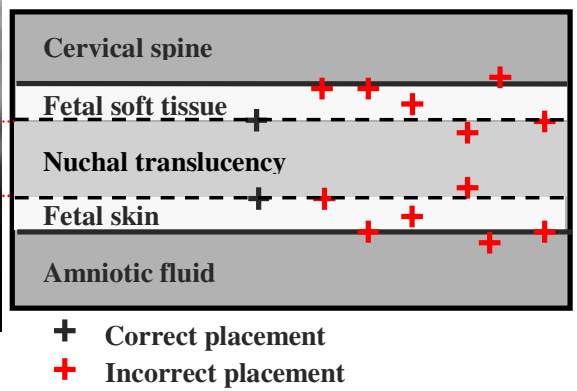

(b)

Fig. 2. Caliper method of measuring NT thickness: (a) ultrasonographic sagittal view of fetus with two calipers; (b) calipers placement on anatomical structures of the fetus

To measure the NT thickness, the calipers are located on the inner borders of the NT edges with none of the horizontal crossbars protruding into the NT layer. The + calipers should be only calipers used for NT measurement. The NT thickness should be measured at its maximum thickness with the placement of the calipers perpendicular to the long axis of the fetus [9]. The ultrasonographic sagittal view of a fetus with two calipers is shown in Fig. 2(a) and Fig. 2(b) illustrates the correct and incorrect placement of the calipers on anatomical structures of the fetus [10].

\subsection{The Region of Interest}

NT images can differ widely because of fetal movement over a scan of several minutes. Fig. 3 shows various NT images corresponding to different fetal positions. In Fig. 3(a) the fetal skin is clearly separate from the amnion, and in Fig. 3(b) there is no clear distinction between fetal skin and the amnion. Fetal images with the head in upside-down or oblique positions are shown in Figs. 3(c) and (d). The ultimate purpose of the identifying the border of the NT layer is to measure the thickness of its widest part. We need to define a region of interest (ROI) which reduces the interference of image boundary and which is adaptable to changes in the fetal head 


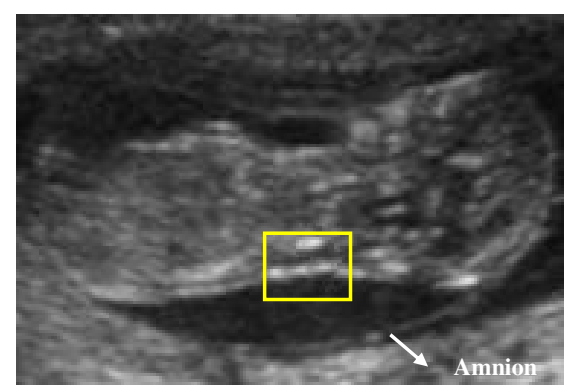

(a)

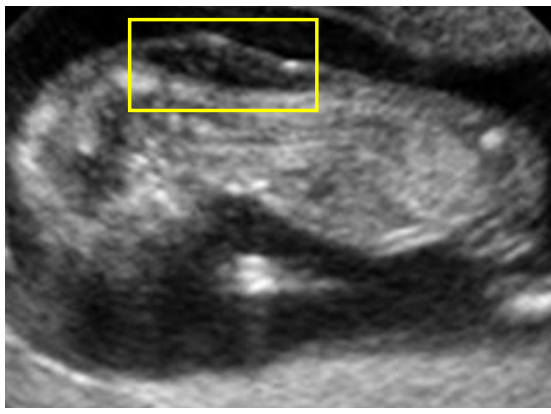

(c)

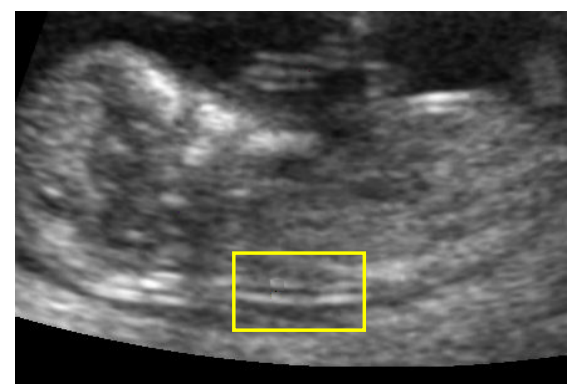

(b)

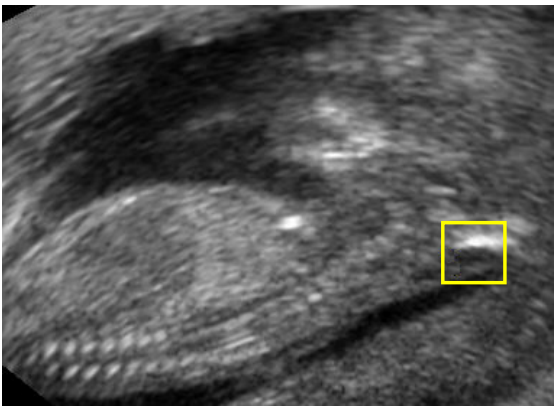

(d)

Fig. 3. NT images of different fetal positions (the rectangle indicates the ROI): (a) fetus image with a clear amniotic edge; (b) a fetus image with an indistinct amnion edge; (c) a fetus in an upside-down position; and (d) a fetus in an oblique position

position and the size of the NT layer. An additional requirement of border detection based on dynamic programming is the choice of start and end-point that enclose the interesting region of the NT layer. We create a rectangular region of interest that includes the widest part of the NT layer in the fetal image by user interaction. By creating the ROI in the correct position, we can avoid interference from speckles and from echo zones $\mathrm{Z} 2$ and $\mathrm{Z} 4$. The coordinates of start and end-point are the top-left and top-right corners of the ROI window.

\subsection{Border Detection}

The accurate segmentation of a border is a very difficult task since ultrasound images usually have a high level of speckle noise and other imaging artifacts due to random scattering. We apply a global optimization approach based on DP to NT border detection. We will now describe this method in detail.

Cost Function. Cost functions are built for each of the borders of the NT layer, which we will call $\mathrm{B} 1$ and $\mathrm{B} 2$. If the $\mathrm{ROI}$ is an $\mathrm{M} \times \mathrm{N}$ rectangle, then all possible borders $B_{N}$ can be considered as polylines with $N$ nodes: 


$$
B_{N}=\left\{p_{1}, p_{2}, \ldots, p_{N-1}, p_{N}\right\},
$$

where $p_{N-1}$ and $p_{N}$ are horizontal neighbors, and $N$ is the horizontal length of a contour line. The cost function $C\left(B_{N}\right)$ is defined as a sum of local costs along a candidate border $B_{N}$ :

$$
C\left(B_{\mathrm{N}}\right)=c_{f}\left(p_{1}\right)+\sum_{i=2}^{N}\left(c_{f}\left(p_{i}\right)+c_{g}\left(p_{i-1}, p_{i}\right)\right) .
$$

At a point $p_{i}$, the local cost is made up of two terms $c_{f}\left(p_{i}\right)$ and $c_{g}\left(p_{i-1}, p_{i}\right)$, which are defined as follows:

$$
\begin{gathered}
c_{f}\left(p_{i}\right)=\sum_{j=1}^{k} w_{j} f_{j}\left(p_{i}\right) \quad(i=1, \ldots, N) \\
c_{g}\left(p_{i-1}, p_{i}\right)=w_{k+1} g\left(p_{i-1}, p_{i}\right) \quad(i=2, \ldots, N),
\end{gathered}
$$

where $f_{j}\left(p_{i}\right)$ are image feature terms, $k$ is the number of image features used, $g\left(p_{i-1}, p_{i}\right)$ is a geometrical force term, and $w_{j}(\mathrm{j}=1,2,3,4)$ is a weighting factor. We use three image features, so $k$ is 3 . The cost terms in the cost function associated with a particular type of border must reflect the characteristics of the image features in its neighborhood and the geometrical form of the border. The cost terms are defined such that a stronger image feature at $p_{i}$ will yield a lower local cost. The desired border corresponds to the $B_{N}$ which minimizes the cost function $C\left(B_{N}\right)$. The weighting factors are determined empirically for each border using the constraint $\mathrm{w}_{1}+\mathrm{w}_{2}+\mathrm{w}_{3}+\mathrm{w}_{4}=1$. Fig. 4 shows a 3D view of the NT layer. Above the NT border B1 (in the y-direction) is a bright and below it is a dark region. The NT border B2 is located above a bright region and below a dark region. From these observations, we can define cost terms that correspond to the characteristics of each border. To be more specific, we will describe the cost terms used in the cost function for B2.

The first term $f_{l}\left(p_{i}\right)$ measures the average intensity of $\mathrm{n}$ (here, $\mathrm{n}=3$ ) pixels below a pixel $p_{i}$ and aims to detect a pixel that belongs to a line above a strong echo zone $\mathrm{Z} 4$. The second term $f_{2}\left(p_{i}\right)$ measures the average intensity of $\mathrm{m}($ here, $\mathrm{m}=2$ ) pixels above a pixel $p_{i}$, and favors a pixel that belongs to a line immediately below a dark NT space Z3. The third term $f_{3}\left(p_{i}\right)$ measures the downward intensity gradient which is expected at the upper edge of an echo zone Z4. The intensity gradient is estimated as vertical intensity slope of the pixel $p_{i}$ using the gradient operator. The final cost term $g\left(p_{i-1}, p_{i}\right)$ is proportional to the square of the difference in vertical distance between the border being estimated and a reference line at node $p_{i}$; this term is designed to ensure border continuity. In the case of $\mathrm{B} 2, g\left(p_{i-1}, p_{i}\right)$ is calculated with a horizontal reference line. In estimating the cost term $g\left(p_{i-1}, p_{i}\right)$ in case of $\mathrm{B} 1$, the reference line is the border $\mathrm{B} 2$ that has already been detected.

Dynamic Programming. Dynamic programming is typically applied to optimization problems, and can be used as an optimal search method for border detection. The 


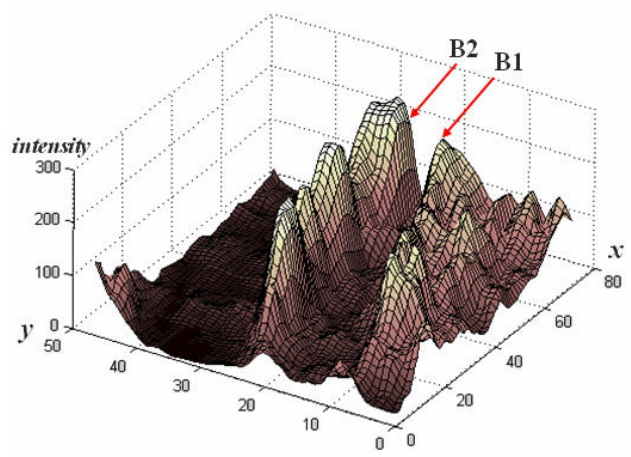

Fig. 4. 3D view of the nuchal translucency layer

optimum border is one that minimizes the given cost function, which is $C\left(B_{N}\right)$ in this case. The search for a solution must be performed globally in the searching region to avoid local minima due to interference patterns such as speckles.

To recast our problem as a DP search, we begin by rewriting Equation (1) in a recursive form as follows:

$$
\begin{aligned}
& C\left(B_{1}\right)=c_{f}\left(B_{1}\right), \\
& C\left(B_{n}\right)=C\left(B_{n-1}\right)+\left(c_{f}\left(p_{n}\right)+c_{g}\left(p_{n-1}, p_{n}\right)\right) \quad(n=2, \ldots, N) .
\end{aligned}
$$

We denote the candidate minima of the cost function of polyline $B_{N}$ as $\tilde{C}\left(B_{n}\right)$. Applying Equation (5), the multistage cost accumulation process can now be expressed as

$$
\begin{aligned}
& \tilde{C}\left(B_{1}\right)=c_{f}\left(B_{1}\right), \\
& \tilde{C}\left(B_{n}\right)=\min _{p_{n-1}}\left\{\tilde{C}\left(B_{n-1}\right)+\left(c_{f}\left(p_{n}\right)+c_{g}\left(p_{n-1}, p_{n}\right)\right)\right\} \quad(n=2, \ldots, N) .
\end{aligned}
$$

To search for the optimum border, costs are accumulated according to Equation (6) and the location of $p_{n-1}$, which defines $\tilde{C}\left(B_{n}\right)$, is stored in a pointer array at each stage $n$. The arrows in Fig. 5(a), point to the pixel in the previous column with the minimum accumulated cost, which then allows the points of the optimal border to be derived by back-tracking.

We will now describe the cost accumulation and back-tracking process in detail within an $\mathrm{M} \times \mathrm{N}$ ROI. A vertical scan window of height $\mathrm{M}$ is used to scan the border from the start to end-point at $N$ horizontal positions. The locations of the start and end-point are the upper-left and upper-right pixels of the ROI respectively, and correspond to the left and right arrows in Fig. 5(b). At each column $n$, a candidate minimum of the cost function is found for each point in that column, and its cost is accumulated. After scanning all the columns, the lowest accumulated cost is located. The position of the minimum cost point becomes the end-point of the border. Then 


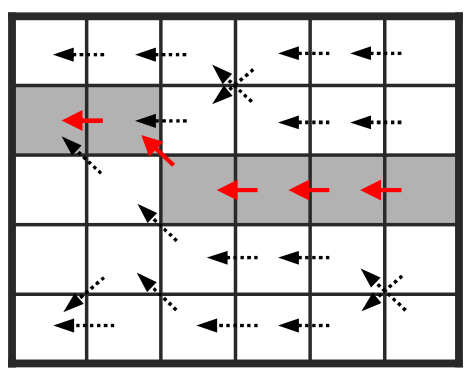

(a)

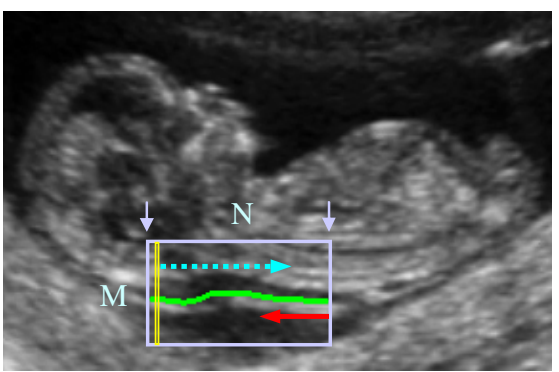

(b)

Fig. 5. Border detection using dynamic programming: (a) the backtracking process, and (b) detecting the border B2 in the NT image

the back-tracking is performed following the pointers until the first column is reached in the ROI. This creates our estimate of the border. B2 can be identified relatively easily since the nearby echogenic zone $\mathrm{Z} 4$ is stronger than echogenic zone $\mathrm{Z} 2$. Therefore we find B2 first. We then search for B1 using a smoothed line of B2 as a reference line, which forms a lower limit in the search for B1.

\subsection{Measurements}

We measure the distance between B1 and B2 using linear regression. The process consists of five steps. In the first step, we calculate mid-points between the upper edge and the lower edge at the 2 right and left neighbors centered on a given value of $x$. The second step is to find the line L1 that bisects the two edges by linear regression. The third step is to find a line L2 that is orthogonal to this bisector L1. The fourth step is to fit lines L3, L4 to B1 and B2 respectively, by linear regression in the range of $x$ that we have previously defined. Finally, we calculate the intersection points between L2 and L3, L4, and then measure the distance between pairs of points. These distances are estimates of the minimum, average and maximum NT thickness.

\section{Implementation and Experimental Results}

The algorithm that we have described in this paper was implemented in Visual C++ on a Pentium IV. We have applied our method to the sagittal section of $2 \mathrm{D}$ and $3 \mathrm{D}$ fetus NT images obtained by transabdominal and transvaginal ultrasonography. Fig. 6 compares the conventional edge-detectors and our method. Fig. 7 shows the resulting borders. The strength of the relationship between automated and manual methods is indicated by the correlation $c_{a, m}$, which is defined as follows: 


$$
c_{a, m}=\frac{\operatorname{Cov}_{a, m}}{\sigma_{a} \sigma_{m}}
$$

where $\operatorname{Cov}_{a, m}$ is the covariance between the automated and manual and $\sigma_{a}$ and $\sigma_{m}$ are the standard deviation of automated and manual measurements, respectively. The means $\left(\mu_{a}, \mu_{m}\right)$ and standard deviation $\left(\sigma_{a}, \sigma_{m}\right)$ for the differences between the

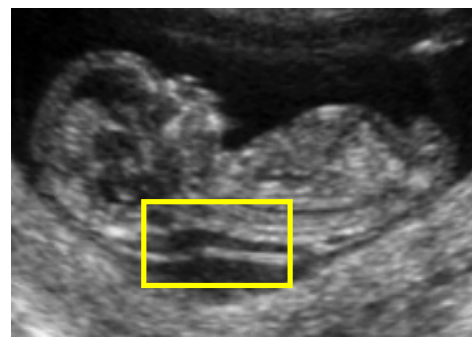

(a)

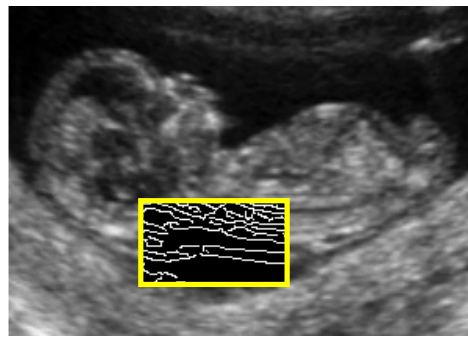

(c)

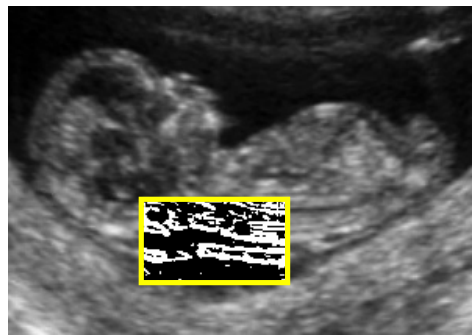

(b)

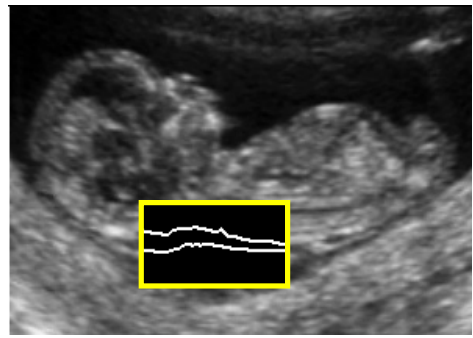

(d)

Fig. 6. Comparison of various edge-detectors: (a) original image; (b) Sobel; (c) Canny; (d) edge-detection using dynamic programming

automatic and manual measurements were calculated for the population $(n=30)$. We calculated the correlation which is defined as accuracy between the automatic and manual measurements of the maximum thickness of the NT layer. The resulting values of the parameters $\mu_{a}, \mu_{m}, \sigma_{a}, \sigma_{m}, c_{a, m}$ are shown in Table 1 .

Table 1. Comparison between automatic and manual methods $(n=30)$

\begin{tabular}{l|c|c|c}
\hline & $\begin{array}{c}\text { Automatic } \\
\mu_{a} \pm \sigma_{a}(\mathrm{~mm})\end{array}$ & $\begin{array}{c}\text { Manual } \\
\mu_{m} \pm \sigma_{m}(\mathrm{~mm})\end{array}$ & $\begin{array}{c}\text { Correlation } \\
c_{a, m}\end{array}$ \\
\hline $\mathrm{NT}_{\max }$ & $2.68 \pm 0.18$ & $2.69 \pm 0.17$ & 0.98 \\
\hline
\end{tabular}



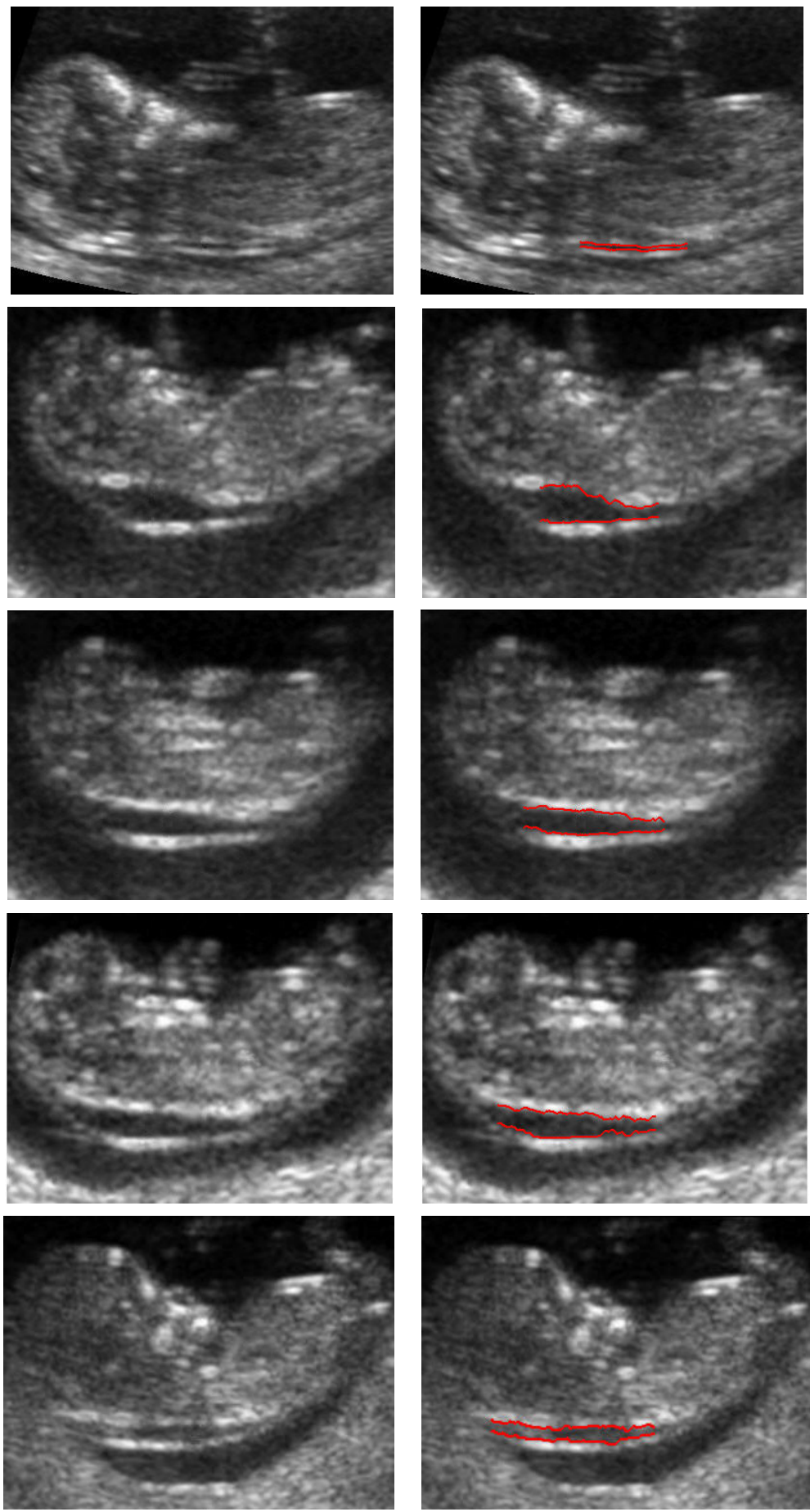

Fig. 7. Experimental results. Left: original image. Right: detecting the border of a fetal NT. 


\section{Conclusions}

We have proposed a method for automated fetal ultrasonic measurements that is based on dynamic programming. Our method detects the borders of the nuchal translucency by constructing a cost function that includes weighted cost terms to represent multiple image features such as intensity, gradient and border continuity. We have obtained superior results to conventional edge-detection procedures that typically consider single image features. In particular, our method has overcome the problem of border continuity which occurs when weak edges are disrupted by the scattering inevitably present in ultrasound images. Our automated measurements were compared with manual measurements, and were found to be equally accurate; and an automated approach reduces the problems of variability and reproducibility that are always present with manual assessments. However, for clinical purposes, some interactive tools may still be needed in order to correct residual detection errors in extremely poor images.

\section{Acknowledgements}

This work is financially supported by the Ministry of Education and Human Resources Development (MOE), the Ministry of Commerce, Industry and Energy (MOCIE) and the Ministry of Labor (MOLAB) through the fostering project of the Lab of Excellency, and by the Korea Institute of Science \& Technology Evaluation and Planning (KISTEP) under the Real Time Molecular Imaging program. We would like to thank the Medison Co. Ltd. for providing ultrasound image datasets for our experiments.

\section{References}

1. W. F. Katherine, A. Toi, S. Salem, K. H. Lisa, D. Chitayat, J. K. Sarah, F. McAuliffe, J. A. Johnson, "Detection of fetal structural abnormalities with US during early pregnancy", RadioGraphics, Vol. 24, 157-174, 2004.

2. R.J. Snijders, P. Noble, N. Sebire, A. Souka, K.H. Nicolaides, "UK multicentre project on assessment of risk of trisomy 21 by maternal age and fetal nuchal translucency thickness at 10-14 weeks of gestation", The Lancet, Vol. 352, 343-346, 1998.

3. A.P. Souka, E. Krampl, S. Bakalis, V. Heath, K.H. Nicolaides, "Outcome of pregnancy in chromosomally normal fetuses with increased nuchal translucency in the first trimester", Ultrasound Obstet. Gynecol. , Vol. 18, 9-17, 2001.

4. F. Bernadino, R. Cardoso, N. Montenegro, J. Bernardes, J. Marques de Sa, "Semiautomated ultrasonographic measurement of fetal nuchal translucency using a computer software tool”, Ultrasound in Med. \& Biol., Vol. 24, No. 1, 51-54, 1998.

5. T. Gustavsson, Q. Liang, I. Wendelhag, J. Wilkstrand, "A dynamic programming procedure for automated ultrasonic measurement of the carotid artery," in Proc. IEEE Computers Cardiology, 297-300, 1994.

6. Q. Liang, I. Wendelhag, J. Wilkstrand, T. Gustavsson, "A multiscale dynamic programming procedure for boundary detection in ultrasonic artery images", IEEE Trans. Med. Imag., Vol. 19, No. 2, 127-142, 2000. 
7. I. Wendelhag, Q. Liang, T. Gustavson, J. Wilkstrand, "A new automated computerized analyzing system simplifies readings and reduces the variability in ultrasound measurement of intima-media thickness", Stroke, Vol. 28, 2195-2200, 1997.

8. M. Bebbington, R.D. Wilson, M.P. Johnson, "Detection of congenital heart disease in the first trimester of pregnancy", Progress in Pediatric Cardiology, Vol. 22, 3-8, 2006.

9. A. Alfred, "Technical Aspects of Nuchal Translucency Measurement", Seminars in Perinatology, Vol. 29, Issue 6, 376-379, 2005.

10. K. Nicolaides, N. Sebire, R. Snijders, "The 11-14 Week Scan: The Diagnosis of Fetal Abnormalities”, Parthenon Publishing Group, New York, 1999. 